\title{
A pseudoneoplastic finding of deep endometriosis: laparoscopic triple segmental bowel resection
}

\author{
Stefano Cosma ${ }^{1}$, Marcello Ceccaroni ${ }^{2}$, Chiara Benedetto ${ }^{1}$ \\ ${ }^{1}$ Department of Gynecology and Obstetrics, Sant'Anna Hospital, University of Turin, Italy \\ ${ }^{2}$ Department of Gynecology and Obstetrics, Gynecologic Oncology Division, International School of Surgical Anatomy, \\ European Gynecology Endoscopy School, Sacred Heart Hospital, Negrar, Verona, Italy
}

Videosurgery Miniinv (e-pub, ahead of print)

DOI: 10.5114/wiitm.2014.41617

\begin{abstract}
Bowel endometriosis affects 3-37\% of patients with endometriosis, involving more frequently the rectum and the rectosigmoid junction. Severe endometriosis with bowel involvement is often refractory to standard medical therapy. For these reasons, surgery for bowel treatment is frequently needed. We report the case of a 36-year-old woman with deep endometriosis of the pelvis, triple segmental bowel involvement (recto-sigma, ileum-cecum, transverse colon) and massive endometriotic ascites with secondary Glisson's capsule inflammation, refractory to medical therapy. A laparoscopic triple segmental bowel resection and complete fertility sparing excision of pelvic endometriotic lesions was performed. At 48 months of follow-up, the woman was asymptomatic, with no evidence of recurrence of disease or ascites. Laparoscopic segmental bowel resection, including multiple section, is feasible in selected symptomatic patients with consequent improved quality of life, morbidity rates similar to those achieved by laparotomy and with a less detrimental effect on fertility.
\end{abstract}

Key words: laparoscopy, endometriosis, bowel resection, ascites.

\section{Introduction}

Deep infiltrating endometriosis is defined as endometriosis infiltrating the peritoneum by more than $5 \mathrm{~mm}$; it is reported in approximately $20 \%$ of all women with endometriosis. Gastrointestinal involvement of endometriosis, the most common location for extra-genital endometriosis, has been reported in $3-37 \%$ of fertile women and the rectum and the recto-sigmoid junction together account for $70-93 \%$ of all intestinal lesions [1]. Small bowel involvement, usually confined to the distal ileum, is less frequent (1-7\%), while cecal endometriosis is rare (0.03\%) [1]. Only one-third of patients with colorectal endometriosis is symptomatic. A bowel obstruction complicates sigmoid endometriosis in $1-10 \%$ of cases [1].

\section{Case report}

A 36-year-old woman referred to our center for worsening dysmenorrhea, dyschezia and epigastric menstrual pain. A diagnostic laparoscopy was performed elsewhere, revealing deep pelvic endometriosis. No bowel surgery was performed and only medical therapy was recommended, without benefit. Furthermore, after 3 years of attempts of spontaneous conception, the patient underwent three unsuccessful cycles of in-vitro fertilization. Abdominal and pelvic ultrasound scan showed pelvic effusion. A tight stenosis of the recto-sigmoid junction did not allow the passage of contrast at the double-contrast barium enema (DCBE) and a computed tomography (CT) scan was performed to investigate the proximal

Address for correspondence:

Stefano Cosma MD, Department of Gynecology and Obstetrics, Sant’Anna Hospital, University of Turin, Via Ventimiglia 3, 10126 Turin,

Italy, phone: +393356643972, e-mail: cosmastefano@libero.it 
colon. A modest increase of markers CA125 (184 UI/ $\mathrm{ml})$ and CA19-9 (85.20 Ul/ml) was observed.

Subsequently, the patient underwent an operative laparoscopy in our center. Copious brownish fluid (4200 $\mathrm{ml}$ ) filled the pelvis, reaching the upper abdomen. The intra-operative cytologic examination of the fluid excluded the presence of neoplastic cells. The Douglas pouch appeared completely obliterated due to the presence of adhesions between the uterus and the rectum-sigmoid junction, where there was an endometriotic implant of about $8 \mathrm{~cm}$. The adnexa were completely surrounded by adhesions. The cecum and the last ileal loop were infiltrated by a nodular mass of about $4 \mathrm{~cm}$ of unknown origin. Similarly, another nodule of $5 \mathrm{~cm}$ was found on the transverse colon, infiltrating the gastrocolic ligament to the edge of the great gastric curvature. Glisson's capsule showed multiple small white lesions. Similar spots were observed on the spleen (Photo 1). An extemporary histological evaluation was carried out on biopsies, showing a pattern suggestive for endometriosis. On intraoperative ultrasound scan, the liver appeared hyperechogenic without focal lesions. Multiple liver biopsies revealed at pathological intraoperative evaluation a framework compatible with reactive capsulitis.

Complete excision of pelvic endometriotic lesions was performed according to a radical, nerve, and fertility sparing surgical technique [2]. The intestinal surgery was performed by a colorectal surgeon after the placement of a fourth trocar on the upper right quadrant of the abdomen (Photo 2).

The left colon was mobilized using an Ultracision Harmonic scalpel (LCS 10; Ethicon Endosurgery, Cincinnati, $\mathrm{OH}, \mathrm{USA}$ ) for dissection. The gastrocolic ligament was opened and the ascending colon was mobilized. Afterwards, the inferior mesenteric vessels were transected and subsequently transection of the rectum was performed. Through a longitudinal supra-umbilical mini-laparotomy sized $4 \mathrm{~cm}$, the trans-

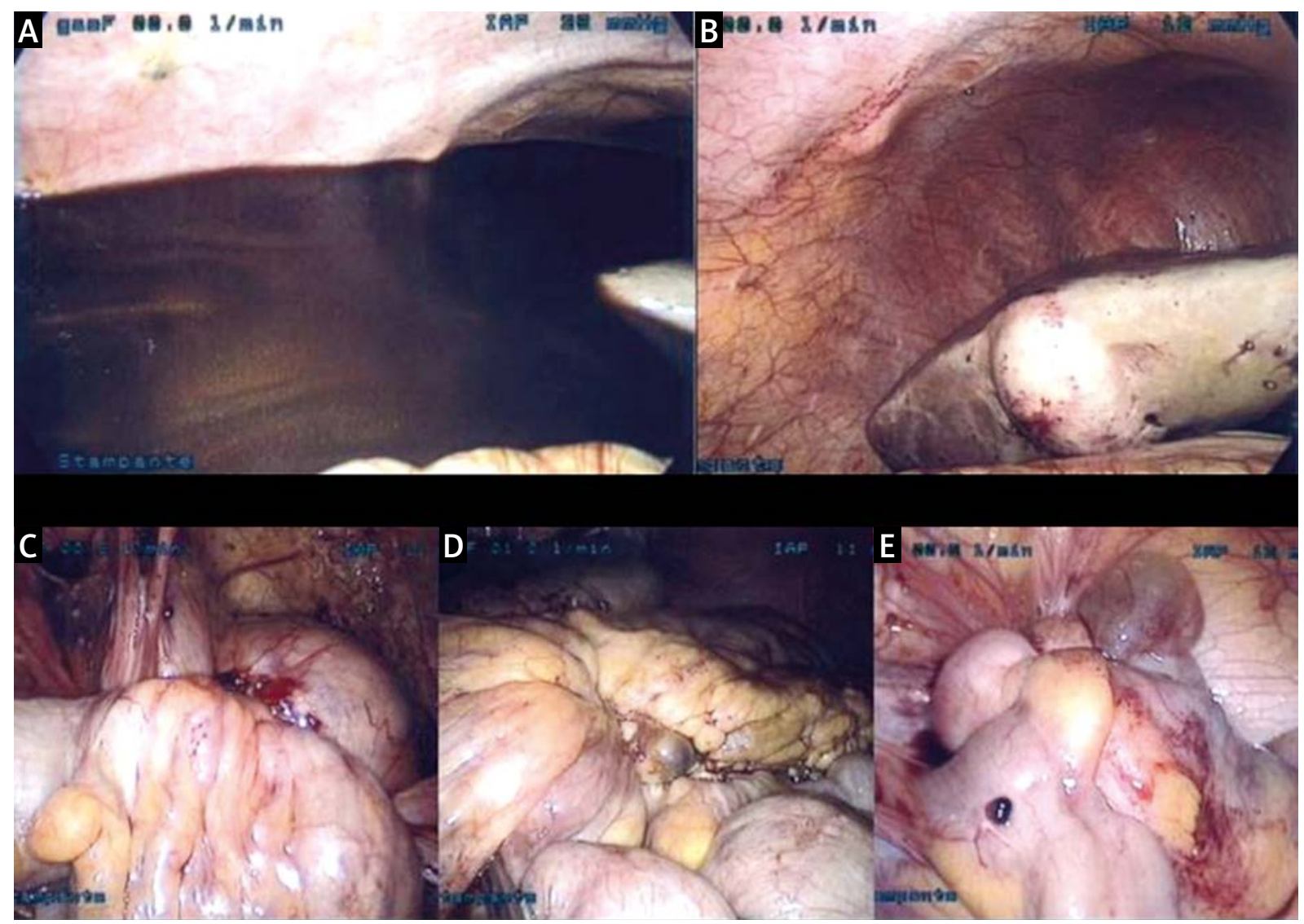

Photo 1. Intraoperative findings. A - Brownish ascites fluid. B - Reactive capsulitis of Glisson's capsule. C - Endometriotic nodule of the rectosigmoid junction. Complete obliteration of Douglas pouch. D - Endometriotic nodule of the transverse colon. $\mathbf{E}$ - Endometriotic nodule of cecum and ileocecal junction 
verse colon was exteriorized and the stenosing part of the bowel was resected; an end-to-end stapled colonic manual anastomosis was performed. Through the same laparotomic incision, the last ileal loop, the cecum and part of the ascending colon were exteriorized. A second bowel resection was performed at this level and an end-to-side stapled mechanical anastomosis (25 mm) was completed; the colonic stump was sutured with GIA 80 (Tyco US Surgical Healthcare Auto Suture). Afterwards, through the same incision, the descending colon, the sigmoid and the rectum were exteriorized to perform a sigmoid-rectal resection. Once pneumoperitoneum was reobtained, a Knight-Griffen end-to-end colorectal anastomosis was completed, after the transanal insertion of a $32 \mathrm{~mm}$ circular end-to-end anastomosis stapling device connected to a circular endoscopic anvil previously positioned. A temporary ileostomy was performed.

The operative time was 480 min with an intraoperative blood loss of $200 \mathrm{ml}$. The patient was discharged on the $8^{\text {th }}$ day.

Forty-two days after surgery, DCBE confirmed that all three anastomoses were intact and ileostomy was converted by an end-to-end stapled anastomosis (Photo 3). Concurrently a laparoscopic second look was performed, showing a recurrent modest amount $(250 \mathrm{ml})$ of ascites and a decrease in the Glisson's capsule inflammation. At 48 months of follow-up, the patient was asymptomatic and no significant pelvic effusion was observed at ultrasound examination. All biochemical markers were normal; liver enzymes were not altered and no signs of malabsorption were observed.

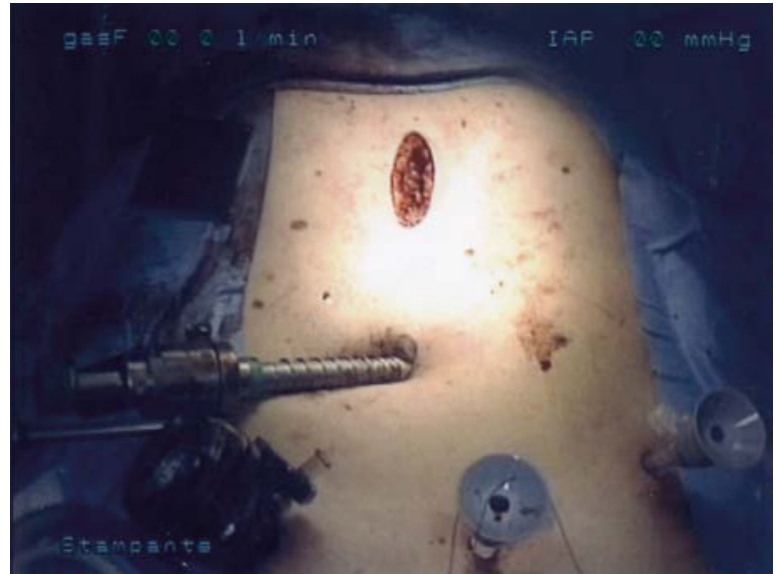

Photo 2. Laparoscopic access 10-mm laparoscope in the standard umbilical position, three 5-mm trocars in suprapubic, left iliac fossa and in the upper right quadrant of the abdomen, one 12-mm trocar in the right iliac fossa and longitudinal supra-umbilical mini-laparotomy

\section{Discussion}

Intestinal endometriosis requiring bowel surgery is present in around one third of cases of severe endometriosis (revised ASRM stage IV) [3]. Surgical resection is required when an obstructive syndrome is present or suspected at the DCBE. The DCBE is considered positive in the presence of a mass effect on the large-bowel wall associated with indentation and ridging of the mucosa. This type of image seems to correspond to an infiltration of the whole muscularis in all cases and to an infiltration reaching the submucosa in $82 \%$ of cases [4]. Due to expressional decrease in both estrogen and progesterone recep-

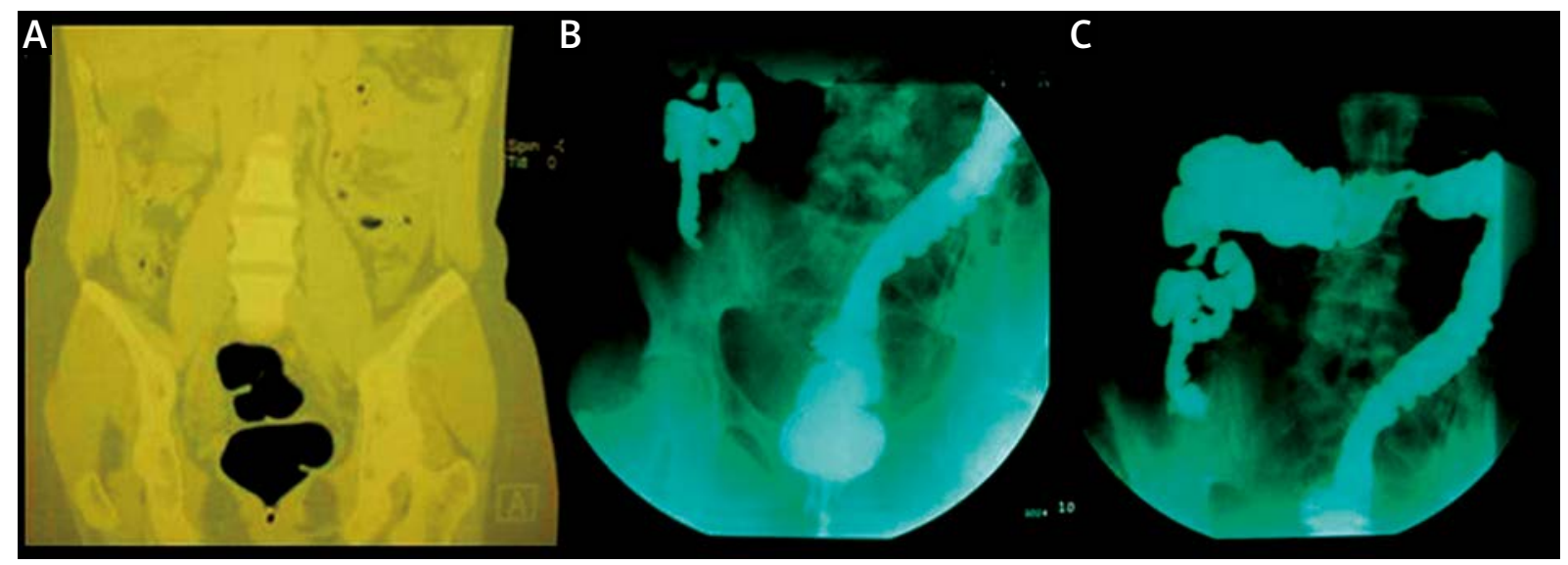

Photo 3. Radiological findings. A - Preoperative CT scan of the colon showed tight stenosis at the rectosigmoid junction. B - Postoperative (42 days later) DCBE showed contrast medium passage through all the anastomoses performed, without leakage 
tors in the ectopic endometrium, the response to hormonal therapy may be compromised [5]. Moreover, endometriotic tissue in the bowel muscularis undergoes muscle cell hyperplasia and fibrosis, becoming resistant to medical treatment.

The extent of rectal surgery has evolved in clinical practice. Brouwer reported a recurrence rate for rectal endometriosis of $22.2 \%$ for dissection of the rectal wall, $5.2 \%$ for wall excision with a circular stapler and $2.2 \%$ for segmental resection [6]. Nodules $>2 \mathrm{~cm}$ or occupying more than one-third of the rectal circumference are suitable for segmental resection. In fact, lesions with circumference involvement $>40 \%$ are associated with an infiltration of the submucous or mucous layer of bowel and with lymph node involvement [7].

Major complications requiring further surgery are reported in $8.3 \%$ of patients who undergo bowel surgery for deep infiltrating endometriosis, whereas recto-vaginal and anastomotic fistulas are described in $3.2 \%$ and $1.1 \%$ of cases [8].

According to the literature, the pregnancy rate after laparoscopic colorectal resection ranges between 34\% and 53.3\% [9], being higher than after laparotomic resection (23.5-39.4\%) [10]. It has been observed that the reproductive outcome improves if bowel resection is performed, leading to conception in $35 \%$ of patients (vs. $21 \%$ of patients without bowel resection), with a shorter interval to obtain a pregnancy (696 vs. 1417 days) [11]. The presence of bowel endometriosis negatively affects fertility, perhaps through obliteration of the pouch of Douglas.

Endometriosis is a heterogeneous disease that ranges in severity as well as manifestations. Involvement of extrapelvic organs is not uncommon. Ectopic endometrium has been found in addition to the gastrointestinal tract, in the urinary system, liver, spleen, diaphragm, pleural cavity, lung and pericardium [12]. Endometriosis can occur under a wide range of conditions mimicking bowel and urinary tract diseases but also malignancy. It is rarely associated with ascites. Less than 50 cases have been reported since the first case described in 1951 [13]. A large volume of ascites may be found and it always presents as a dark brown or hemorrhagic fluid. Sometimes an associated endometrioma is described but also normal ovaries at inspection are reported [14]. The cytological diagnosis of peritoneal endometriosis can be difficult since non-specific hemosiderin-laden or hemofuscin-laden macrophages may be the only cells identified [15]. Its pathophysiology is unclear but it can result from the rupture of endometriomas or, rarely, from hepatic involvement by cystic endometriosis. The hypothesis that endometrial cells shedding into the peritoneal cavity may irritate the peritoneum, thereby leading to ascites, is also widely accepted. The effectiveness of ovarian suppression therapy and bilateral ovariectomy is consistent with the hypothesis that the ovary is the origin of the massive ascites (similar to the Meigs syndrome). Whether ascites is due to endometriosis is debated, although the deep brown color suggests a causal relationship [16].

\section{Conclusions}

Therefore the suspicion of endometriosis is justified in young fertile women with suggestive clinical history, even in the presence of diagnostic and intraoperative findings mimicking neoplastic disease, and makes reasonable, in a referral center, an initial minimally invasive approach also in these borderline cases.

A laparoscopic approach to severe pelvic endometriosis with segmental bowel resection is feasible and achieves the same results in terms of outcome and quality of life compared with traditional open surgery with a lower complication rate [10]. In women with bowel symptomatic endometriosis, laparoscopic colorectal resection can be accomplished with a lower impact on fertility, compared to the laparotomic approach [10].

This kind of surgery is not free of severe complications, so it should be performed only in selected patients and in reference centers.

\section{References}

1. Bergqvist A. Different types of extragenital endometriosis: a review. Gynecol Endocrinol 1993; 7: 207-21.

2. Landi S, Ceccaroni M, Perutelli A, et al. Laparoscopic nerve-sparing complete excision of deep endometriosis: is it feasible? Hum Reprod 2006; 21: 774-81.

3. Minelli L, Ceccaroni M, Ruffo G, et al. Laparoscopic conservative surgery for stage IV symptomatic endometriosis: short-term surgical complications. Fertil Steril 2010; 94: 1218-22.

4. Chapron C, Vieira M, Chopin N, et al. Accuracy of rectal endoscopic ultrasonography and magnetic resonance imaging in the diagnosis of rectal involvement for patient presenting with deeply infiltrating endometriosis. Ultrasound Obstet Gynecol 2004; 24: 175-9.

5. Guo T, Gu C, Feng C, et al. Endoureterotomy is not a sufficient treatment for intrinsic ureteral endometriosis. Videosurgery Miniinv 2013; 8: 187-91. 
6. Brouwer R, Rodney J. Woods Rectal endometriosis: results of radical excision and review of published work. ANZ J Surg 2007; 77: 562-71.

7. Abrão MS, Podgaec S, Dias JA Jr, et al. Endometriosis lesions that compromise the rectum deeper than the inner muscularis layer have more than $40 \%$ of the circumference of the rectum affected by the disease. J Minim Invasive Gynecol 2008; 15: 774-5.

8. Ruffo G, Scopelliti F, Scioscia M, et al. Laparoscopic colorectal resection for deep infiltrating endometriosis: analysis of 436 cases. Surg Endosc 2010; 24: 63-7.

9. Mohr C, Nezhat FR, Nezhat CH, et al. Fertility considerations in laparoscopic treatment of infiltrative bowel endometriosis. JSLS 2005; 9: 16-24.

10. Daraï E, Dubernard G, Coutant C, eta I. Randomized trial of laparoscopically assisted versus open colorectal resection for en dometriosis: morbidity, symptoms, quality of life, and fertility. Ann Surg 2010; 251: 1018-23.

11. Stepniewska A, Pomini P, Bruni F, et al. Laparoscopic treatment of bowel endometriosis in infertile women. Hum Reprod 2009; 24: 1619-25.

12. Ceccaroni M, Roviglione G, Rosenberg P, et al. Pericardial, pleural and diaphragmatic endometriosis in association with pelvic peritoneal and bowel endometriosis: a case report and review of the literature. Videosurgery Miniinv 2012; 7: 122-31.

13. Brews A. Endometriosis including endometriosis of the diaphragm and Meigs' syndrome. Proc R Soc Med 1951; 47: 461.

14. Cheng MH, Yen MS, Chao KC, et al. Differential diagnosis of gynecologic organ-related diseases in women presenting with ascites. Taiwan J Obstet Gynecol 2008; 47: 384-90.

15. Goumenou A, Matalliotakis I, Mahutte N, Koumantakis E. Endometriosis mimicking advanced ovarian cancer. Fertil Steril 2006; 86: 219.

16. Ussia A, Betsas G, Corona R, et al. Pathophysiology of cyclic hemorrhagic ascites and endometriosis. J Minim Invasive Gynecol 2008; 15: 677-81.

Received: 26.09.2013, accepted: 5.11.2013. 